BULl, AUSTRAL, MATH. SOC.

$47 \mathrm{H} 10$

VOL. 34 (1986) 107-117.

\title{
CONVEX SETS, FIXED POINTS, VARIATIONAL AND MINIMAX INEQUALITIES
}

\author{
TZU-Chu LIN
}

\begin{abstract}
Recently, Ky Fan extended his will known lemma (which is an extension of the classical theorem of Knaster, Kuratowski and Mazurkiewica) to the noncompact case. Using this result, another interesting lemma of $F$ an is generalized in this paper. As applications of our theorem, we obtain a generalization of Browder's variational inequality and derive Fan's other recent results directly from our theorem. Also, in this paper, we give a slight extension recent results of $\mathrm{K}$. K. Tan, which themselves are generalizations of many well-known results on minimax and variational inequalities.
\end{abstract}

\section{Introduction.}

In 1961, Fan [4, Lemma 1] gave an extension of the classical Knaster-Kuratowski-Mazurkiewicz theorem [8] to an arbitrary Hausdorff topological vector space. Since then, this result has been widely used in nonlinear functional analysis, and is known as Fan's Lemma or K-K-M-Fans' Theorem (see [3]). Recently, Fan [7, Theorem 4] extended his well known lemma to the noncompact case. In this paper, we first use Fan's Theorem

Received 7 October 1985.

Copyright Clearance Centre, Inc. Serial-fee code: 0004-9727/86 $\$ A 2.00+0.00$. 
[7, Theorem 4] to obtain a generalization of another interesting lemma of Fan [4, Lemma 4]. As applications of our theorem, we obtain a generalization of Browder's variational inequality [2, Theorem 2] and directly from our theorem derive Theorems 7, 8 of Fan [7]. We also prove a slight generalization of Theorems 1, 2 of Tan [11] and other results contained therin, which itself is a generalization of many well-known results on minimax and variational inequalities.

We first state some definitions.

DEFINITION. Let $X$ be a nonempty convex subset of a Hausdorff topological vector space $E . A$ real-valued function $f$ on $X$ is said to be

(i) lower semicontinuous if for each $t$ the set $\{x \in X \mid f(x) \leq t\}$ is closed;

(ii) convex if for $x, y$ in $X$ and $0 \leq r \leq 1$ we have $f((1-r) y+r x) \leq(1-r) f(y)+r f(x)$;

(iii) quasi-concave if for each $t$ the set $\{x \in X \mid f(x)>t\}$ is convex or empty.

We will denote by $\operatorname{co}\left\{x_{1}, \ldots, x_{n}\right\}$, the convex full of any finite subset $\left\{x_{1}, \ldots, x_{n}\right\}$ of $E$.

\section{Main Results.}

We use the following Lemma [7] to pxove Theorem 1.

LEMMA 1. (Fan [7, Theorem 4]). In a Hausdorff topological vector space, let $Y$ be a convex set and $\emptyset \neq X \subset Y$. For each $x \in X$, let $F(x)$ be a relatively closed subset of $Y$ such that the convex hull of every finite subset $\left\{x_{1}, x_{2}, \ldots, x_{n}\right\}$ of $X$ is contained in the corresponding vonion $\bigcup_{i=1}^{n} F\left(x_{i}\right)$. If there is a nonempty subset $x_{0}$ of $X$ such that the intersection $\cap_{x \in X_{0}}^{n} F(x)$ is compact and $X_{0}$ is contained in a compact convex subset of $Y$, then $n E(x) \neq \emptyset$. $x \in X$ Remark 1. Note that $\cap_{x \in X_{0}}^{n} F(x)$ cannot be empty since, if it were, 
taking $A(x)$ to be the complement of $F(x)$ in $Y$ and applying Lemma 1 of [7] we would have a finite subset of $X_{0}$ whose convex hull contained a point outside the union of the corresponding $F(x)$ 's.

THEOREM 1. Let $X$ be a nonempty convex subset of a Housdorff topological vector space $E$. Let $A \subset X \times X$ be a subset such that

(a) for each $x \in X$, the set $\{y \in X \mid(x, y) \in A\}$ is closed in $X$;

(b) for each $y \in X$, the set $\{x \in X \mid(x, y) \notin A\}$ is convex or empty;

(c) $(x, x) \in A$ for each $x \in X$;

(d) $X$ has a nonempty compact convex subset $X_{0}$ such that the set $B=\left\{y \in X \mid(x, y) \in A\right.$ for all $\left.x \in X_{0}\right\}$ is compact.

Then there exists a point $y_{0} \in B$ such that $X \times\left\{y_{0}\right\} \subset A$.

Proof. For each $x \in X$, let $F(x)=\{y \in X \mid(x, y) \in A\}$. By assumption (a), $F(x)$ is closed in $X$. By assumptions (b), (c), $\operatorname{co}\left\{x_{1}, \ldots, x_{n}\right\} c$ $n$ $\bigcup_{i=1}^{u} F\left(x_{i}\right)$ for any finite subset $\left\{x_{1}, \ldots, x_{n}\right\}$ of $X$. Indeed, let $z=\sum_{i=1}^{n} \alpha_{i} x_{i}, \sum_{i=1}^{n} \alpha_{i}=1, \alpha_{i} \geq 0, i=1, \ldots, n$. If $z \notin \underset{i=1}{n} F\left(x_{i}\right)$, then $\left(x_{i}, z\right) \notin A$ for $i=1, \ldots, n$. By assumption (b) applied to this $z$, the set $\{x \in X \mid(x, z) \notin A\}$ is convex. Therefore $(z, z) \notin A$, which contradicts (c). By assumption (d), the intersection $n E(x)$ is $x \in X_{0}$

contained in $B$ and is compact. By Lemma 1, there exists a point $y_{0} \in \underset{x \in X}{\cap} F(x)$, which means $X \times\left\{y_{0}\right\} \subset A$.

Remark 2. (i) Condition (d) of Theorem 1 can be replaced by the following condition:

(dI) let $X_{0}$ be a nonempty compact convex subset of $X$, and $K$ a nonempty compact subset of $X$. If for every $y \in X \backslash K$, there is a point $x \in X_{0}$ such that $(x, y) \notin A$.

We remark that, under the assumption (a) of the theorem, (dl) is a special case of (d). Indeed, by (dl), the set $\left\{y \in X \mid\left(f x \in X_{0}\right)((x, y) \in A)\right\} \subset K$. By 
(a), the set $\left\{y \in X \mid\left(\forall x \in X_{0}\right)((x, y) \in A)\right\}$ is closed, and is compact.

From the above remark, we see that, under condition (dl), our conclusion will be: there exists a point $y_{0} \in K$ such that $X \times\left\{y_{0}\right\} \subset A$.

(ii) As in Remark 1, $B$ is necessarily non-empty.

(iii) If $K=X$, then condition (dl) is automatically satisfied.

COROLLARY 1. (Fan [4, Lerma 4]). Let $X$ be a nonempty compact convex subset of a Housdorff topological vector space $E$. Let $A \subset X \times X$ be a subset such that the conditions (a), (b), (c) of Theorem 1 are satisfied.

Then there exists a point $y_{0} \in X$ such that $X \times\left\{y_{0}\right\} \subset A$.

Proof. From Theorem 1 and Remark 2.

Now we will see some applications of Theorem 1. We first obtain a generalization of a variational inequality of Browder [R, Theorem 2].

THEOREM 2. Let $X$ be a nonempty convex subset of a locally convex Hausdorff topological vector space $E, T$ a continuous mapping of $X$ into $E^{*}$.

(d) If $X$ has a nonempty compact convex subset $X_{0}$ such that the set $B=\{y \in X \mid(\forall x \in X)((T y, y-x) \geq 0)\}$ is compact, then there exists a point $y_{0} \in B$ such that $\left(T y_{0}, y_{0}-x\right) \geq 0$ for alz $x \in X$.

Proof. Let

$$
A=\{(x, y) \in X \times X \mid(T y, y-x) \geq 0\} .
$$

since $T$ is continuous, the set $\{y \in X \mid(x, y) \in A\}$ is closed in $X$ for each $x \in X$. It is clearly that $(x, x) \in A$ for each $x \in X$, and the set $\{x \in X \mid(x, y) \notin A\}=\{x \in X \mid(T y, y-x)<0\}$ is convex or empty for each $y \in X$. BY Theorem 1, there exists a point $y_{0} \in B$ such that $X \times\left\{y_{0}\right\} \subset A$, that is $\left(T y_{0}, y_{0}-x\right) \geq 0$ for all $x \in X$.

Remark 3. (i) Condition (d) of Theorem 2 can be replaced by the following condition:

(d2) let $X_{0}$ be a nonempty compact convex subset of $X$, and $K$ a nonempty compact subset of $X$. If for every $y \in X \backslash K$, there is a point $x \in X_{0}$ such that $(T y, y-x)<0$. 
(ii) We have the same remark as Remark 2 (ii), (iii). We will not repeat this statement for other remarks.

COROLLARY 2. (Browder [2, Theorem 2]). Let $X$ be a nonempty compact convex subset of a locally convex Hausdorff topological vector space $E, T$ a continuous mapping of $X$ into $E^{*}$. Then there exists a point $y_{0} \epsilon X$ such that $\left(T_{0}, y_{0}-x\right) \geq 0$ for alz $x \in X$.

Proof. This follows from Theorem 2 and Remark 3.

Now we derive the following theorems of Fan ([7, Theorem 7,8$]$ ) directly from our Theorem 1.

THEOREM 3. [7, Theorem 7]. Let $X$ be a nonempty convex set in a normed vector space $E$, and let $f: X \rightarrow E$ be a continuous map.

(d) If $X$ has a nonempty compact convex subset $X_{0}$ such that the set $B=\left\{y \in X\left|\left(\forall x \in X_{0}\right)\right||x-f(y)\|\geq|| y-f(y)\|)\right\}$ is compact, then there exists a point $y_{0} \in B$ such that

$$
\left\|y_{0}-f\left(y_{0}\right)\right\|=\min _{x \in X}\left\|x-f\left(y_{0}\right)\right\| \text {. }
$$

(In particular, if $f\left(y_{0}\right) \in X$, then $y_{0}$ is a fixed point of $f$ ).

Proof. Let

$$
A=\{(x, y) \in X \times X \mid\|x-f(y)\| \geq\|y-f(y)\|\} .
$$

Since $f$ is continuous, the set $\{y \in X \mid(x, y) \in A\}$ is closed in $X$ for each $x \in X$. It is clear that $(x, x) \in A$ for each $x \in X$, and the set $\{x \in X \mid(x, y) \notin A\}=\{x \in X|||x-f(y)\|<\| y-f(y)| \mid\}$ is convex or empty. By Theorem 1, there exists a point $y_{0} \in B$ such that $X \times\left\{y_{0}\right\} \subset A$,

$$
\left\|y_{0}-f\left(y_{0}\right)\right\|=\min _{x \in X} \mid x-f\left(y_{0}\right) \| .
$$

Remark.4. (i) The condition (d) of Theorem 3 can be replaced by the following condition:

(d3) Let $X_{0}$ be a nonempty compact convex subset of $X$, and $K$ a nonempty compact subset of $X$, such that for every $y \in X \backslash K$, there is a point $x \in X_{0}$ such that $\|x-f(y)\|<\|y-f(y)\|$.

Under the condition (d3), we can conclude that $y_{0} \in K$ such that 


$$
\left\|y_{0}-f\left(y_{0}\right)\right\|=\underset{x \in X}{\min \left\|x-f\left(y_{0}\right)\right\| .}
$$

Actually, Fan [7] proved Theorem 3 under the condition (d3).

(ii) If $K=X$, this reduces to Fan [5, Theorem 2] . Other generalizations of this result for a closed convex subset $K=X$ in Hilbert space were obtained by the author $[9,10]$ and also for a closed ball in a Banach space [9].

THEOREM 4. (Fan [7, Theorem 8]). Let $X$ be a nonempty poracompact convex set in a Housdorff topological vector space. Let $\Omega$ ba a nonempty convex set (that is every convex combination of any two functions in $\Omega$ is in $\Omega$, of Zower semi-continuous convex functions on $X$. Let $S$ be a subset of $X \times \Omega$ such that:

(a) For each fixed $\phi \in \Omega$, the section $S(\phi)=\{x \in X \mid(x, \phi) \in S\}$ is open in $x$.

(b) For any fixed $x \in X$, the section $S(x)=\{\phi \epsilon \Omega \mid(x, \phi) \in S\}$ is convex and nonempty.

Then either there exists $\left(y_{1}, \phi_{1}\right) \in S$ satisfying

$$
y_{1} \in X \text { and } \phi_{1}\left(y_{1}\right)=\operatorname{Min}_{x \in X} \phi_{1}(x) \text {; }
$$

or for any nonempty compact convex subset $X_{0}$ of $X$ and any compact subset $K$ of $X$, there exists $\left(y_{2}, \phi_{2}\right) \in S$ satisfying

$$
y_{2} \in X \backslash K \text { and } \phi_{2}\left(y_{2}\right) \leq \phi_{2}(x) \text { for all } x \in X_{0} \text {. }
$$

Proof. Our proof is a modification of Fan [7. Theorem 8]. By (b), for each $z \in X$, there is a $\phi_{z} \in \Omega$ such that $\left(z, \phi_{z}\right) \in S$. By (a), $\left\{S\left(\phi_{z}\right) \mid z \in X\right\}$ is an open cover of the paracompact space $X$. Let $\left\{\alpha_{z} \mid z \in X\right\}$ be a continuous partition of unity subordinate to this open cover. Thus, for each $z \in X, \alpha_{z}$ is a non-negative real continuous function on $X$, with its support supp $\alpha_{z} \subset S\left(\phi_{z}\right)$. The family $\left\{\right.$ supp $\left.\alpha_{z} \mid z \in X\right\}$ is a locally finite closed cover of $X ;$ and $\sum_{z \in X} \alpha_{z}(x)=1$ for all $x \in X$, define $\psi(x)$ by

$$
\psi(x)=\sum_{z \in X} \alpha_{z}(x) \phi_{z}
$$


which is a convex combination of a finite number of $\phi_{z}{ }^{\prime} s$. As $\Omega$ is convex, we have $\psi(x) \in \Omega$ for each $x \in X$. If $x, z$ in $X$ are such that $\alpha_{z}(x)>0$, then $x \in \operatorname{supp} \alpha_{z} \subset S\left(\phi_{z}\right)$ and therefore $\phi_{z} \in S(x)$. By (b), we have $\psi(x) \in S(x)$ for all $x \in X$.

Now, define

$$
A=\{(x, y) \in X \times X \mid \psi(y)(y) \leq \psi(y)(x)\} .
$$

Since $\alpha_{z}$ is continuous anā non-negative on $X$ and $\phi_{z}$ is lower semicontinuous on $X$, then for each fixed $x \in X$, the function

$$
h(x, y)=\psi(y)(y)-\psi(y)(x)=\sum_{z \in X} \alpha_{z}(y) \phi_{z}(y)-\sum_{z \in X} \alpha_{z}(y) \phi_{z}(x)
$$

is also a lower semi-continuous function of $y$ on $X$. Therefore the set

$$
\{y \in X \mid(x, y) \in A\}=\{y \in X \mid \psi(y)(y) \leq \psi(y)(x)\}
$$

is closed in $X$ for each $x \in X$. It is clear that $(x, x) \in A$ for all $x \in X$ and the set $\{x \in X \mid(x, y) \notin A\}$ is convex or empty for each $y \in X$ (since $\psi(y) \in \Omega$ and $\psi(y)$ is a convex function on $X)$. By Theorem 1 and Remark 2, either there exists $y_{1} \in X$ such that $X \times\left\{y_{1}\right\} \subset A$; or for any non-empty compact convex subset $X_{0}$ of $X$ and any compact subset $K$ of $X$, there exists $y_{2} \in X \backslash K$ such that $\left(x, y_{2}\right) \in A$ for all $x \in X_{0}$. We take $\phi_{1}=\psi\left(y_{1}\right)$ in the first case, and $\phi_{2}=\psi\left(y_{2}\right)$ in the second case. In the first case, $X \times\left\{y_{1}\right\} \in A$, this means

$$
\phi_{1}\left(y_{1}\right)=\operatorname{Min}_{x \in X} \phi_{1}(x) .
$$

since $\psi\left(y_{1}\right) \in S\left(y_{1}\right),\left(y_{1}, \phi_{1}\right) \in S$. In the second case, $\left(x, y_{2}\right) \in A$ for all $x \in X_{0}$, it implies that

$$
\phi_{2}\left(y_{2}\right) \leq \phi_{2}(x) \quad \text { for all } x \in X_{0} \text {. }
$$

This completes the proof.

Now, we will use Lemma 1 to prove the following minimax inequalities.

THEOREM 5. Let $X$ be a nonempty convex set in a Housdorff topological vector space $E$. Let $f$ and $g$ be two real-valued function on $X \times X$ hoving the following properties:

(a) $g(x, y) \leq f(x, y)$ for all $(x, y) \in X \times X$ and $f(x, x) \leq 0$ for alz $x \in X$; 
(b) for each fixed $X \in X, g(x, y)$ is a lower semicontinuous function $y$ on $x$;

(c) for each fixed $y \in X$, the set $\{x \in X \mid f(x, y)>0\}$ is convex or empty;

(d) $X$ has a nonempty compact convex subset $X_{0}$ such that the set $B=\left\{y \in X \mid g(x, y) \leq 0\right.$ for azz $\left.x \in X_{0}\right\}$ is compact.

Then there exists a point $y_{0} \in B$ such that $g\left(x, y_{0}\right) \leq 0$ for all $x \in X$.

Proof. For each $x \in X$, let

$$
\begin{aligned}
& G(x)=\{y \in X \mid g(x, y) \leq 0\}, \\
& F(x)=\{y \in X \mid f(x, y) \leq 0\} .
\end{aligned}
$$

By (b), $G(x)$ is closed in $X$. From (a), (c), we have

$$
\operatorname{co}\left\{x_{1}, \ldots, x_{n}\right\} \subset \bigcup_{i=1}^{n} E\left(x_{i}\right)
$$

for any finite subset $\left\{x_{1}, \ldots, x_{n}\right\}$ of $X$. Indeed, if $z=\sum_{i=1}^{n} \alpha_{i} x_{i}$, $\sum_{i=1}^{n} \alpha_{i}=1, \alpha_{i} \geq 0$, and $z \notin \bigcup_{i=1}^{n} F\left(x_{i}\right)$, then $\left(f\left(x_{i}\right), z\right)>0, i=1, \ldots, n$. By (c), $f(z, z)>0$, which contradicts the assumption (a). By (a) $F(x) \subset G(x)$. Then $\operatorname{co}\left\{x_{1}, \ldots, x_{n}\right\} \subset \cup_{i=1}^{n} G\left(x_{i}\right)$. Since $\operatorname{n}_{x \in X_{0}} G(x)$ is a closed subset of the compact set $B, \cap G(x)$ is compact. By Lemma 1 , $x \in X_{0}$

there exists a point $y_{0} \in \underset{x \in X}{n} G(x)$, which means $g\left(x, y_{0}\right) \leq 0$ for all $x \in X$.

Remark 5. Condition (d) of Theorem 5 can be replaced by the following condition:

(d5) let $X_{0}$ be a nonempty compact convex subset of $X$, and $K$ a nonempty compact subset of $X$. If for every $y \in X \backslash K$, there is a point $x \in X_{0}$ such that $g(x, y)>0$. Then our conclusion is that there exists a point $y_{0} \in K$ such that

$$
g\left(x, y_{0}\right) \leq 0 \text { for all } x \in X
$$


COROLLARY 3. (Tan [11, Theorem 1]). Let $X, E, f$, and $g$ be the same as in Theorem 5 and satisfy conditions $(a),(b),(c)$ and (d') there exists a nonempty compact convex subset $K$ of $X$ such that for each $y \in X \backslash K$, there exists a point $x \in K$ with $g(x, y)>0$.

Then there exists a point $y_{0} \in K$ such that $g\left(x, y_{0}\right) \leq 0$ for all $x \in X$.

Proof. Take $X_{0}=K$ in (d5).

Remark 6. If $f=g$ in Corollary 3, this result is due to Allen [1, Theorem 2].

THEOREM 6. Let $X$ be a nonempty convex set in a Hausdorff topological vector space. Let $f$ and $g$ be two real-valued function on $X \times X$ hoving the following properties:

(a) $g(x, y) \leq f(x, y)$ for all $(x, y) \in X \times X$,

(b) for each fixed $x \in X, g(x, y)$ is a lower semicontinuous function of $y$ on $x$,

(c) for each fixed $y \in X, f(x, y)$ is a quasi-concove fronction of $x$ on $X$,

(d) if $X$ has a non-empty compact convex subset $X_{0}$ such that the set $B=\left\{y \in X \mid\left(\forall x \in X_{0}\right)(g(x, y) \leq t)\right\}$ is compact, if

$$
t=\sup _{x \in X} f(x, x)<+\infty \text {. }
$$

Then the minimax inequality

$$
\min _{y \in B} \sup _{x \in X} g(x, y) \leq \sup _{X \in X} f(x, x)
$$

holds.

Proof. Without loss of generality, we can assume that $t=\sup _{x \in X} f(x, x)<+\infty$.

Applying Theorem 5 to

$$
f_{1}(x, y)=f(x, y)=t, g_{1}(x, y)=g(x, y)-t,
$$

the proof is complete.

Remark 7. Condition (d) of Theorem 6 can be replaced by the following condition: 
(d6) let $X_{0}$ be a nonempty compact convex subset of $X$, and $K$ a nonempty compact subset of $X$. If for every $y \in X \backslash K$, there is a point $x \in X_{0}$ such that

$$
g(x, y)>t \text { if } t=\sup _{x \in X} f(x, x)<+\infty
$$

then our conclusion is that the minimax inequality

holds.

$$
\min _{y \in K} \sup _{x \in X} g(x, y) \leq \sup _{x \in X} f(x, x)
$$

COROLLARY 4. (Tan [11, Theorem 2]). Let $X, f, g$ be the same as Theorem 6 and satisfy conditions (a), (b), (c) and

(d') there exists a nonempty compact convex subset $K$ of $X$ such that for all $y \in X \backslash K$, there exists a point $x \in K$ with $g(x, y)>t$ if $t=\sup _{x \in X} f(x, x)<\infty$.

Then the minimax inequality

$$
\min _{y \in K} \sup _{x \in X} g(x, y) \leq \sup _{x \in X} f(x, y)
$$

holds.

Proof. Take $X_{O}=K$ in $(d 6)$

Remark 8. If $K=X$, the result is due to Yen [72, Theorem 1]. If $K=X, f=g$, the result is due to Fan [6].

Remark 9. Tan [11] applied corollaries 3, 4 to obtain some generalizations of variational inequalities $([11$, Theorem 3,5$])$ and fixed point theorems ([11, Theorem 6-8]). Applying our Theorems 5, 6 and using the same arguments as $\operatorname{Tan}[11]$, we can obtain a slight generalization of the corresponding Theorems $3,5,6,7,8$ of Tan [11], just like we did for Theorems 5 and 6 and Remarks 5, 7 .

\section{References}

[1] G. Allen, "Variational inequalities, complementarity problems, and duality theorems", J. Math. AnaZ. App Z. 58 (1977), 1-10

[2] F.E. Browder, "The fixed point theory of multi-valued mappings in topological vestor spaces", Math. Ann. 177 (1968), 283-301.

[3] J. Dugundji and A. Granas, Fixed point theory, Vol. I (PWN-Polish Scientific Publishers, Warszawa, 1982). 
[4] K. Fan, "A generalization of Tychonoff's fixed point theorem", Math. Ann. 142 (1961), 305-310.

[5] K. Fan, "Extensions of two fixed point theorens of F. E. Bowder", Math. 2. 112 (1969), 234-240.

[6] K. Fan, "A minimax inequality and applications", Inequalities III (ed. ). Shisha, Academic Press, New York, 1972), 103-113.

[7] K. Fan, "Some properties of convex sets related to fixed point theorems", Math. Ann. 266 (1984), 519-537.

[8] B. Knaster, C. Kuratowski and S. Mazurkiewicz, Ein Beweis des Fixpunktsatzes fur n-dimensionale Simplexe", Fund. Math. 14 (1929), 132-137.

[9] T. C. Lin, "A note on a theorem of Ky Fan", Canad. Math. Buzz. 22 (1979), 513-515.

[10] T. C. Lin and C. L. Yen, "Applications of the proximity map to fixed point theorems in Hilbert space", J. Approx. Theory (to appear).

[11] K. K. Tan, "Comparison theorems on minimax inequalities, variational inequalities, and fixed point theorems", $J$. London Math. Soc. 28 (1983), 555-562.

[12] C. L. Yen, "A minimax inequality and its applications to variational inequalities", Pacific J. Math. 97 (1981), 477-481.

Department of Mathematical Sciences

The University of Wisconsin-Milwaukee

P.o. Box 413

Milwaukee, Wisconsin 53201

U.S.A.

Added in Proof. I would like to thank Professor Sehie Park of Seoul National University (Korea) for calling my attention to the recent paper of $\mathrm{KY} F$ an [7], when we met at the International Conference on Approximation Theory held in Newfoundland, Canada, August 1984. 